\title{
PREMATURE AORTIC STIFFNESS IN SYSTEMIC LUPUS ERYTHEMATOSUS BY TRANSESOPHAGEAL ECHOCARDIOGRAPHY
}

\author{
Carlos A. Roldan, M.D., Joseph Joson, M.D., Clifford R. Qualls, Ph.D., Janeen Sharrar, RN, \\ and Wilmer L. Sibbitt Jr, M.D. \\ Department of Medicine, Divisions of Cardiology and Rheumatology, University of New Mexico \\ School of Medicine. Albuquerque New Mexico, USA.
}

\section{SUMMARY}

To assess aortic stiffness by transesophageal echocardiography (TEE) and to determine its clinical predictors and relation to age, blood pressure, renal function, and atherosclerosis, 50 patients with systemic lupus erythematosus (SLE), 94\% women, with a mean age of $38 \pm 12$ years and 22 age and gender matched healthy controls underwent clinical and laboratory evaluations and multiplane TEE to assess stiffness, intima-media thickness (IMT), and plaques of the proximal, mid, and distal descending thoracic aorta. At each level and overall aortic stiffness by the pressure-strain elastic modulus was higher in patients than in controls after adjusting for age (overall, $8.25 \pm 4.13$ versus $6.1 \pm 2.5$ Pascal units, $p=0.01)$. Patients had higher aortic stiffness than controls after adjusting both groups to the same mean age, blood pressure, creatinine, and aortic IMT ( $\mathrm{p}=$ 0.005). Neither IMT nor plaques were predictors of aortic stiffness. Moreover, normotensive patients, those without aortic plaques, and non-smokers had higher stiffness than controls (all $\mathrm{p}$ $<0.05)$. Age of SLE diagnosis, non-neurologic damage score, and mean arterial pressure during TEE were the only independent predictors of aortic stiffness (all $p \quad \unlhd$ (02). Thus, aortic stiffness may be a primary form of premature functional vasculopathy in SLE.

\section{Keywords}

aortic stiffness; systemic lupus erythematosus; transesophageal echocardiography; atherosclerosis

\section{INTRODUCTION}

\begin{abstract}
Patients with systemic lupus erythematosus (SLE) have a high prevalence of hypertension and premature coronary and carotid atherosclerosis that substantially increases their morbidity and mortality (1-7). Patients with SLE also develop arterial stiffness, but it is uncertain if arterial stiffness occurs independently of age, hypertension, renal function, or atherosclerosis $(8,9)$. In addition, aortic stiffness may be causally related to the development of hypertension and premature aortic atherosclerosis (10). Therefore, this study was designed with 3 objectives: 1 ) to directly assess aortic stiffness using transesophageal echocardiography (TEE) in young SLE patients as compared to age and gender matched healthy controls; 2) to determine the clinical predictors of aortic stiffness; and 3) to determine if aortic stiffness occurs independently of age, blood pressure, renal function, and aortic atherosclerosis.
\end{abstract}

Corresponding Author: Carlos A. Roldan, M.D., Professor of Medicine, University of New Mexico School of Medicine, Cardiology Division 5-ACC, MSC 10 5550, 1 University of New Mexico, Albuquerque New Mexico 87131-0001, Phone: (505) 272-6020, croldan@salud.unm.edu. 


\section{PATIENTS AND METHODS}

\section{Study populations}

Fifty consecutive patients with diagnosis of SLE according to criteria by the American College of Rheumatology, 46 women, with a mean age of $38 \pm 12$ years (range, 18-60) and 22 healthy volunteers, 19 women, with a mean age of $34 \pm 12$ years (range, 18-57) agreed to participate in the study. Patients with SLE were recruited from a well-characterized population of about 200 patients between 18 to 60 years old regularly followed at the Rheumatology Clinics of the University of New Mexico Health Sciences Center. This study protocol was approved by the Institutional Review Board and all participating subjects signed an approved informed consent.

\section{Clinical and laboratory evaluations}

Patients and controls underwent clinical and laboratory evaluations including specific parameters of inflammation (Table 1). Also, SLE patients were further characterized with regard to their disease duration, activity, severity, therapy, and serology including antiphospholipid antibodies (Table 2).

\section{Transesophageal Echocardiography}

All subjects underwent multiplane TEE with Philips I-E33 imaging systems using a $7 \mathrm{MHz}$ transducer with an axial resolution of approximately $0.1 \mathrm{~mm}$. At a low depth $(3-4 \mathrm{~cm})$ and using a narrow sector scan to improve image resolution, two-dimensional guided M-mode images were used to assess aortic systolic and diastolic diameters and intima-media thickness (IMT) of the aortic anterior wall at the proximal (25-30 cm from the incisors), mid $(30-35 \mathrm{~cm})$ and distal descending thoracic aorta $(35-40 \mathrm{~cm})$. Aortic diameters were measured from the short and long axis views during end-systole and end-diastole (peak to end of $T$ wave and after $P$ wave on the electrocardiogram, respectively) Figure 1). At each aortic level, 3-6 measurements of diameters and IMT were averaged. Far field decreased resolution and common off axis views precluded accurate assessment of diameters and IMT of ascending aorta and arch. All studies were codified, digitally stored, and those of patients and controls were randomly intermixed. Also, all studies were interpreted and measured off line using electronic calipers by experienced observers blinded to subjects' all clinical data. To further avoid interpretation bias, one observer (JJ) assessed aortic diameters and a second observer (CAR) assessed IMT and plaques. Finally, to assess intraobserver reliability 26 randomly selected studies (from 16 patients and 10 controls) had repeated measurements of aortic diameters.

\section{Blood pressures}

During TEE and at each aortic level multiple blood pressure measurements were obtained from the brachial artery using an automatic Dash 2000 sphygmomanometer. Blood pressures were matched in time with aortic measurements.

\section{Assessment of aortic stiffness and atherosclerosis}

Aortic stiffness was assessed with the Pressure-Strain Elastic Modulus (PSEM as $=[\boldsymbol{k}(\mathbf{s B P}$ $-\boldsymbol{d B P}) /(\boldsymbol{s} \boldsymbol{D}-\boldsymbol{d D} / \boldsymbol{d D})] / 100$ where $\mathbf{k}=133.3$ is a conversion factor from $\mathrm{mmHg}$ to Pascal units, $\mathbf{S B P}=$ systolic blood pressure, $\mathbf{d B P}=$ diastolic blood pressure, $\mathbf{s D}=$ systolic diameter, and $\mathbf{d D}=$ diastolic diameter (11). $\boldsymbol{P S E M}$ is a well-established and validated parameter of arterial stiffness that defines the amount of force (pulse pressure) required to distend a vessel. This parameter allows assessment of aortic stiffness independently of IMT or plaques. Atherosclerosis was defined as aortic plaques manifested as $>50 \%$ focal or protruding wall thickening as compared with surrounding walls $(4,5,12)$. 


\section{Statistical Analysis}

Student's $t$ test or Wilcoxon rank-sum test (for normally or non-normally distributed data) and Fisher's exact test were used for comparison of continuous and categorical variables among groups, respectively. Analysis of aortic stiffness was performed with repeated measures ANOVA where the 3 aortic locations of measurements constitute a repeated factor and SLE patients versus control subjects constitute the group factor. The "best" multivariate models were obtained by stepwise regression and verified by "all subsets" regression for the stiffness parameter on the candidate predictors (those with $\mathrm{p}<0.05$ in univariate analysis). The effect size for a predictor in both univariate and multivariate models are reported as correlations or standardized betas (regression coefficients of the standardized outcome and predictor variables). To further determine that aortic stiffness in SLE patients occurs independently of age, blood pressure, renal function, and atherosclerosis we adjusted by multivariate regression each individual's PSEM at each aortic location to the same mean age (37 years old), arterial blood pressure $(86 \mathrm{mmHg})$, creatinine $(0.80 \mathrm{mg} / \mathrm{dl})$, and IMT $(0.81$ $\mathrm{mm})$. These means are for the pooled data of patients and controls. A two-tail $\mathrm{p}$ value $<0.05$ was considered significant.

\section{RESULTS}

\section{Clinical characteristics of patients and controls}

SLE patients and controls were similar in age, gender, and body mass index. However, SLE patients had higher ambulatory blood pressures, more atherogenic risk factors, higher creatinine (only 3 patients had values $>1 \mathrm{mg} / \mathrm{dL}$ ) and lower hematocrit, platelets, and albumin than controls (all p $₫ \mathbf{0} .04$, Table 1 ). Clinical, therapy, and laboratory data specific to SLE patients are delineated in Table 2.

\section{Blood pressures, aortic diameters, aortic intima-media thickness and plaques in patients and controls}

During TEE, patients had higher systolic, diastolic, and mean arterial blood pressures than controls $(123 \pm 18,71 \pm 16$, and $89 \pm 16 \mathrm{mmHg}$ versus $113 \pm 12,64 \pm 12$, and $81 \pm 11$ $\mathrm{mmHg}$, respectively, all $\mathrm{p} \unlhd \mathbf{\Delta} .05$ ). However, pulse pressures were similar in both groups (51 \pm 13 versus $49 \pm 12 \mathrm{mmHg}$, respectively, $\mathrm{p}=0.39$ ). Although, patients and controls had similar systolic and diastolic aortic diameters $(1.99 \pm 0.26$ and $1.81 \pm 0.28$ versus $1.94 \pm$ 0.22 and $1.74 \pm 0.24 \mathrm{~cm}$, respectively, both $\searrow 0.25$ ), the differential diameter was lower in patients than in controls $(0.18 \pm 0.05$ versus $0.20 \pm 0.04 \mathrm{~cm}$, respectively, $\mathrm{p}=0.03)$. Also, patients had higher IMT values and more aortic plaques than controls $(0.87 \pm 0.35$ versus $0.68 \pm 0.18 \mathrm{~mm}$ and $28 \%$ versus $0 \%$, respectively, both $\mathrm{p} \unlhd 0.02$ ). The percent error in the measurement of systolic and diastolic diameters at the proximal, mid, and distal aorta were $6 \%$ and $6 \%, 2 \%$ and $3 \%$, and $4 \%$ and $6 \%$, respectively.

\section{Aortic stiffness in patients and controls}

Stiffness was higher in all SLE patients, in normotensive SLE patients (sBP $\leq 135$ and dPB $\$ 85 \mathrm{mmHg}$ ), in those without aortic plaques, and in non-smokers as compared to controls (all $\mathrm{p}<0.05)$ (Table 3). Also, aortic stiffness was higher in patients with than without pre or hypertension on TEE and aortic plaques $(12.3 \pm 5.6$ versus $7.4 \pm 3.2$ and $9.4 \pm 4.3$ versus 7.8 \pm 4 units, $p=0.03$ and $p=0.26$, respectively). By repeated measures ANOVA and adjusted for age, aortic stiffness was higher in patients than in controls $(\mathrm{p}=0.02)$ and progressively increased from proximal to distal aorta $(\mathrm{p}=0.04)$ (Figures 2). In addition, after adjustment of both groups to the same mean age, arterial blood pressure, creatinine, and IMT, SLE patients still had significantly higher aortic stiffness as compared to controls $(p=0.005)$ with a linear trend up of stiffness from proximal to distal aorta $(\mathrm{p}=0.04)$ (Figure 3$)$. 


\section{Predictors of aortic stiffness in patients with SLE (Table 4}

By univariate analyses including all clinical, therapy, and laboratory variables listed in Tables $1 \& 2$, age of SLE diagnosis, mean arterial blood pressure during TEE, nonneurologic and total SLE damage scores, C3a, IgG anticardiolipin antibodies, total cholesterol and platelets levels were predictors of aortic stiffness (all p $\mathbf{\$}$.05). However, aortic IMT, aortic plaques, smoking, and any atherogenic risk factor, were not predictors. By multivariate analyses, age of SLE diagnosis, non-neurologic SLE damage score, and mean arterial blood pressure during TEE (expectedly since blood pressure is a factor in the calculation of stiffness) were the only independent predictors of aortic stiffness (all $\mathrm{p}<0.02$ ).

\section{DISCUSSION}

\section{Major Findings}

There are three major findings in this study: 1) aortic stiffness is higher in young SLE patients as compared to matched controls after adjusting for age; 2) in SLE patients, age of SLE diagnosis, non-neurologic damage score, and mean arterial blood pressure are the only independent predictors of aortic stiffness; and 3) aortic stiffness in SLE occurs independently of age, renal function, blood pressure, and aortic atherosclerosis. To our knowledge this is the first TEE series in young SLE patients to directly demonstrate the occurrence of aortic stiffness independently of age, blood pressure, renal function, atherogenic risk factors, and early aortic atherosclerosis.

This study findings suggest that the later in life SLE is diagnosed the higher the likelihood that untreated immune-mediated inflammation leads to aortic stiffness. However, since the age of SLE diagnosis is known but not the age of SLE onset, it is also possible that the later the age of SLE diagnosis is the higher the likelihood that age related aortic stiffness occurs. Therefore, delayed SLE diagnosis and older age at diagnosis play a role in the pathogenesis of aortic stiffness in SLE.

Also in this study, normotensive SLE patients had higher aortic stiffness than controls. Morover, aortic stiffness was higher in patients with than without hypertension. These findings support that once SLE related aortic stiffness occurs, hypertension may follow and result in a perpetuating vicious cycle of progressive large vessel vasculopathy $(3,8,9,10-15)$. However, SLE related hypertension may also lead to aortic stiffness, which then also perpetuates hypertension. In this study, SLE patients had higher non-hypertensive range blood pressures than controls, $14 \%$ of patients had hypertension and $28 \%$ were either hypertensive or on vasodilator therapy. This is a significantly higher prevalence of hypertension in young SLE women as compared to that of a similar age and gender general population $(\sim 5 \%)(16)$. Also, hypertension was not related to the low prevalence of renal disease $(6 \%)$ and postmenopausal status $(15 \%)$ nor body mass index since patients and controls had similar values. Similarly high prevalence rates (29\%-36\%) of hypertension have been reported in several series of premenopausal SLE women $(2,3,15,17)$. Therefore, the putative mechanisms of hypertension in SLE such as inflammatory cytokine mediated endothelial dysfunction, hyperactivity of the renal angiotensin system resulting in increased production of angiotensin II and endothelin-1 (both potent vasoconstrictors), increased oxidative stress, steroid therapy, and insulin resistance may all actually contribute to aortic stiffness and then to hypertension or both simultaneously $(2,14,20)$.

In addition, in this study SLE patients without aortic plaques had higher aortic stiffness than controls. Although not statistically significant, aortic stiffness was also higher in patients with than without aortic plaques. These findings also support that aortic stiffness in SLE may be an important pathogenic or exacerbating factor for aortic atherosclerosis, which then sets up a self perpetuating pathologic cycle $(10,13-15)$. In this study, $28 \%$ of patients had 
aortic plaques ( $\mathrm{p}=0.004$ versus controls), a similar prevalence to that of coronary ( $31 \%)$ and carotid $(37 \%)$ atherosclerosis reported in other series $(4,15,18)$.

Therefore, aortic stiffness in SLE may lead or contribute to the development of hypertension, which begets stiffness and both may accelerate aortic atherosclerosis.

\section{Comparison with previous studies}

Although no previous series have directly assessed aortic stiffness by TEE in SLE, several series using arterial tonometry have demonstrated either aortic or peripheral arterial stiffness manifested by an increased carotid to femoral pulse wave velocity (PWV) $(5,8,9)$. Selzer et al (3), in a non-controlled study of 214 SLE women without clinical cardiovascular disease with a mean age of $45.2 \pm 10.5$ years (included patients $>60$ years old) demonstrated that aortic stiffness by PWV was associated with older age, higher systolic blood pressure, higher $\mathrm{C} 3$ levels, lower white blood cell count, higher insulin levels, and renal disease. Bjarnegråd $\mathrm{N}$, et al (8), in 27 SLE women (52 to 68 years old) and 27 controls demonstrated that carotid to femoral PWV was higher in SLE women than in controls after correction for mean arterial pressure and body mass index. Also, aortic PWV was positively associated to C-reactive protein and complement factor 3. Finally, Yildiz M, et al (9), demonstrated higher carotid-femoral PWV in 24 premenopausal SLE women as compared to 24 age- and sex-matched controls. Aortic stiffness was correlated with age, body mass index, waist--tohip ratio, heart rate and blood pressure. Unlike our study, these series did not demonstrate the occurrence of aortic stiffness independently of age, blood pressure, renal function, and aortic atherosclerosis.

\section{Limitations of the study}

An underestimation of aortic stiffness may have occurred because this study population only represents about $25 \%$ of patients regularly followed at our institution, thus, patients with severe disease may be underrepresented; the low proportion (6\%) of patients with renal dysfunction studied; and the assessment of IMT and plaques of the aortic posterior wall was limited and therefore aortic atherosclerosis may have been underestimated. In contrast, the small healthy control group may have led to an overestimation of the independent effect of SLE on aortic stiffness. Also an independent effect of blood pressure on aortic stiffness could only be partially ascertained because assessment of aortic stiffness includes blood pressure as a factor. However, when patients' and controls' mean arterial blood pressure was adjusted to the same normal value or normotensive patients were compared with controls, aortic stiffness was still significantly higher in patients than in controls. Finally, although the specific effect of different SLE therapies on aortic stiffness was not assessed, they were not predictors in the univariate and multivariate analyses.

\section{Clinical implications}

This study may have several clinical implications: 1) aortic stiffness may be a primary form of premature functional vasculopathy in SLE (19), 2) SLE-associated chronic immunemediated inflammation and damage to the arterial wall cause aortic stiffness, which then may lead to or exacerbate hypertension, and then to atherosclerosis (20); 3) earlier diagnosis and aggressive anti-inflammatory therapy of SLE has the potential to prevent the development and progression of aortic stiffness, hypertension, and atherosclerosis $(20,21)$; and 4) statin, angiotensin converting enzyme inhibitor, and antiplatelet therapy may also have primary and secondary preventive effects due to SLE-associated high prevalence of dyslipidemia, endothelial dysfunction, hypertension, vascular stiffness, and atherosclerosis $(22,23)$. These interventions have the potential to decrease the prevalence and incidence of cardiac and cerebrovascular diseases in SLE and their associated high morbidity and mortality. However, a large prospective controlled cross-sectional and longitudinal study is 
necessary to define better the short and long term prognosis of aortic stiffness on cardiac and cerebrovascular outcomes in SLE.

\section{Acknowledgments}

Grant support: This research was funded by the grant RO1-HL04722-01-A3, NIH-NHLBI and 5M02 RR00997.

\section{REFERENCES}

1. Becker-Merok A, Nossent J. Prevalence, predictors and outcome of vascular damage in systemic lupus erythematosus. Lupus. 2009; 18:508-515. [PubMed: 19395452]

2. Urowitz MB, Gladman D, Ibañez D, et al. Clinical manifestations and coronary artery disease risk factors at diagnosis of systemic lupus erythematosus: data from an international inception cohort. Lupus. 2007; 16:731-735. [PubMed: 17728367]

3. Selzer F, Sutton-Tyrrell K, Fitzgerald SG, et al. Comparison of risk factors for vascular disease in the carotid artery and aorta in women with systemic lupus erythematosus. Arthritis Rheum. 2004; 50:151-159. [PubMed: 14730611]

4. Asanuma Y, Oeser A, Shintani AK, et al. Premature coronary artery atherosclerosis in systemic lupus erythematosus. N Engl J Med. 2003; 349:2407-2415. [PubMed: 14681506]

5. Roman MJ, Shanker BA, Davis A, et al. Prevalence and correlates of accelerated atherosclerosis in systemic lupus erythematosus. N Engl J Med. 2003; 349:2399-2406. [PubMed: 14681505]

6. Ippolito A, Petri M. An update on mortality in systemic lupus erythematosus. Clin Exp Rheumatol. 2008; (5 Suppl 51):S72-S79. [PubMed: 19026147]

7. Nossent J, Cikes N, Kiss E, et al. Current causes of death in systemic lupus erythematosus in Europe, 2000--2004: relation to disease activity and damage accrual. Lupus. 2007; 16:309-317. [PubMed: 17576731]

8. Bjarnegråd N, Bengtsson C, Brodszki J, Sturfelt G, Nived O, Länne T. Increased aortic pulse wave velocity in middle aged women with systemic lupus erythematosus. Lupus. 2006; 15:644-650. [PubMed: 17120590]

9. Yildiz M, Yildiz BS, Soy M, Tutkan H. Impairment of arterial distensibility in premenopausal women with systemic lupus erythematosus. Kardiol Pol. 2008; 66:1194-1199. [PubMed: 19105096]

10. Laurent S, Boutouyrie P, Asmar R, et al. Aortic stiffness is an independent predictor of all-cause and cardiovascular mortality in hypertensive patients. Hypertension. 2001; 37:1236-1241. [PubMed: 11358934]

11. Godia EC, Madhok R, Pittman J, et al. Carotid artery distensibility: a reliability study. J Ultrasound Med. 2007; 26(9):1157-1165. [PubMed: 17715309]

12. Manzi S, Selzer F, Sutton-Tyrrell K, et al. Prevalence and risk factors of carotid plaque in women with systemic lupus erythematosus. Arthritis Rheum. 1999; 42:151-160.

13. Selzer F, Sutton-Tyrrell K, Fitzgerald S, Tracy R, Kuller L, Manzi S. Vascular stiffness in women with systemic lupus erythematosus. Hypertension. 2001; 37:1075-1082. [PubMed: 11304506]

14. Ryan MJ. The pathophysiology of hypertension in systemic lupus erythematosus. Am J Physiol Regul Integr Comp Physiol. 2009; 296:R1258-R1267. [PubMed: 19158408]

15. Protogerou A, Blacher J, Stergiou GS, Achimastos A, Safar ME. Blood response under chronic antihypertensive drug therapy: the role of aortic stiffness in the REASON (Preterax in regression of arterial stiffness in a controlled double blind) study. J Am Coll Cardiol. 2009; 53:445-454. [PubMed: 19179203]

16. Cutler JA, Sorlie PD, Wolz M, Thom T, Fields LE, Roccella EJ. Trends in Hypertension Prevalence, Awareness, Treatment, and Control Rates in United States Adults Between 1988-1994 and 1999-2004. Hypertension. 2008; 52:818-827. [PubMed: 18852389]

17. Zhang CY, Lu LJ, Li FH, et al. Evaluation of Risk Factors That Contribute to High Prevalence of Premature Atherosclerosis in Chinese Premenopausal Systemic Lupus Erythematosus Patients. J Clin Rheumatol. 2009; 15:111-116. [PubMed: 19300290] 
18. Cacciapaglia F, Zardi EM, Coppolino G, et al. Stiffness parameters, intima-media thickness and early atherosclerosis in systemic lupus erythematosus patients. Lupus. 2009; 18:249-256. [PubMed: 19213864]

19. El-Magadmi M, Bodill H, Ahmad Y, et al. Systemic lupus erythematosus: an independent risk factor for endothelial dysfunction in women. Circulation. 2004; 110:339-404.

20. Booth AD, Wallace S, McEniery CM, Yasmin, Brown J, Jayne DR, Wilkinson IB. Inflammation and arterial stiffness in systemic vasculitis: a model of vascular inflammation. Arthritis Rheum. 2004; 50:581-588. [PubMed: 14872502]

21. Kabakov AE, Tertov VV, Saenko VA, Poverenny AM, Orekhov AN. The atherogenic effect of lupus sera: systemic lupus erythematosus-derived immune complexes stimulate the accumulation of cholesterol in cultured smooth muscle cells from human aorta. Clin Immunol Immunopathol. 1992; 63:214-220. [PubMed: 1623641]

22. Avalos I, Chung CP, Oeser A, et al. Aspirin therapy and thromboxane biosynthesis in systemic lupus erythematosus. Lupus. 2007; 16:981-986. [PubMed: 18042592]

23. Ferreira GA, Navarro TP, Telles RW, Andrade LE, Sato EI. Atorvastatin therapy improves endothelial-dependent vasodilation in patients with systemic lupus erythematosus: an 8 weeks controlled trial. Rheumatology (Oxford). 2007; 46:1560-1565. [PubMed: 17693444] 


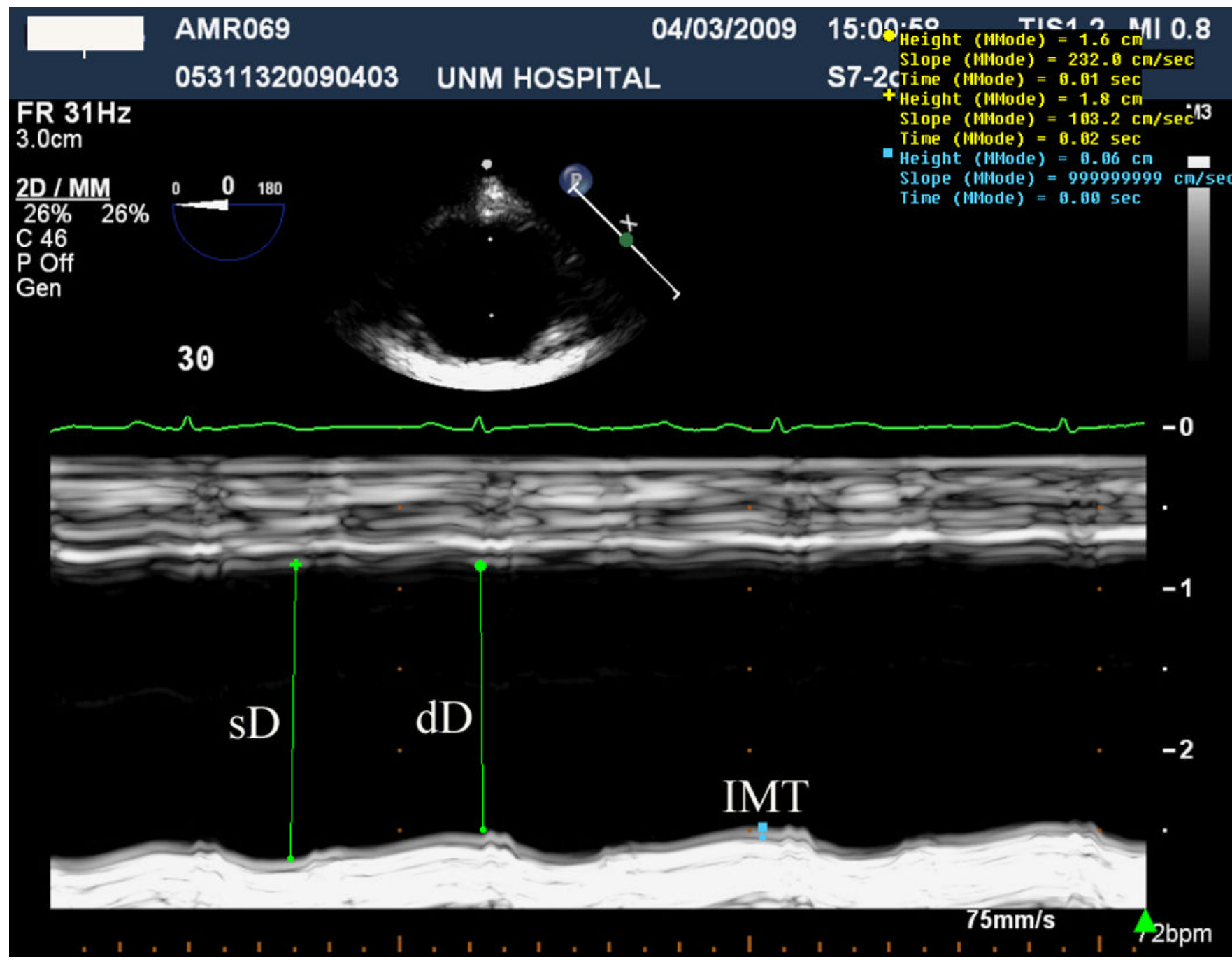

Figure 1. Measurement of Aortic Diameters and Intima-media Thickness

This TEE short axis two-dimensional guided M-mode image of the proximal descending thoracic aorta in a patient with SLE demonstrates the measurements of the aortic systolic diameter $(\mathrm{sD})$ at the peak of the $\mathrm{T}$ wave on the electrocardiogram and measurement of the diastolic diameter (dD) and aortic intima-media thickness (IMT) during end-diastole (after the $\mathrm{P}$ wave on the electrocardiogram). The systolic and diastolic diameters and IMT measured $1.8 \mathrm{~cm}, 1.6 \mathrm{~cm}$, and $0.6 \mathrm{~mm}$, respectively. 
10
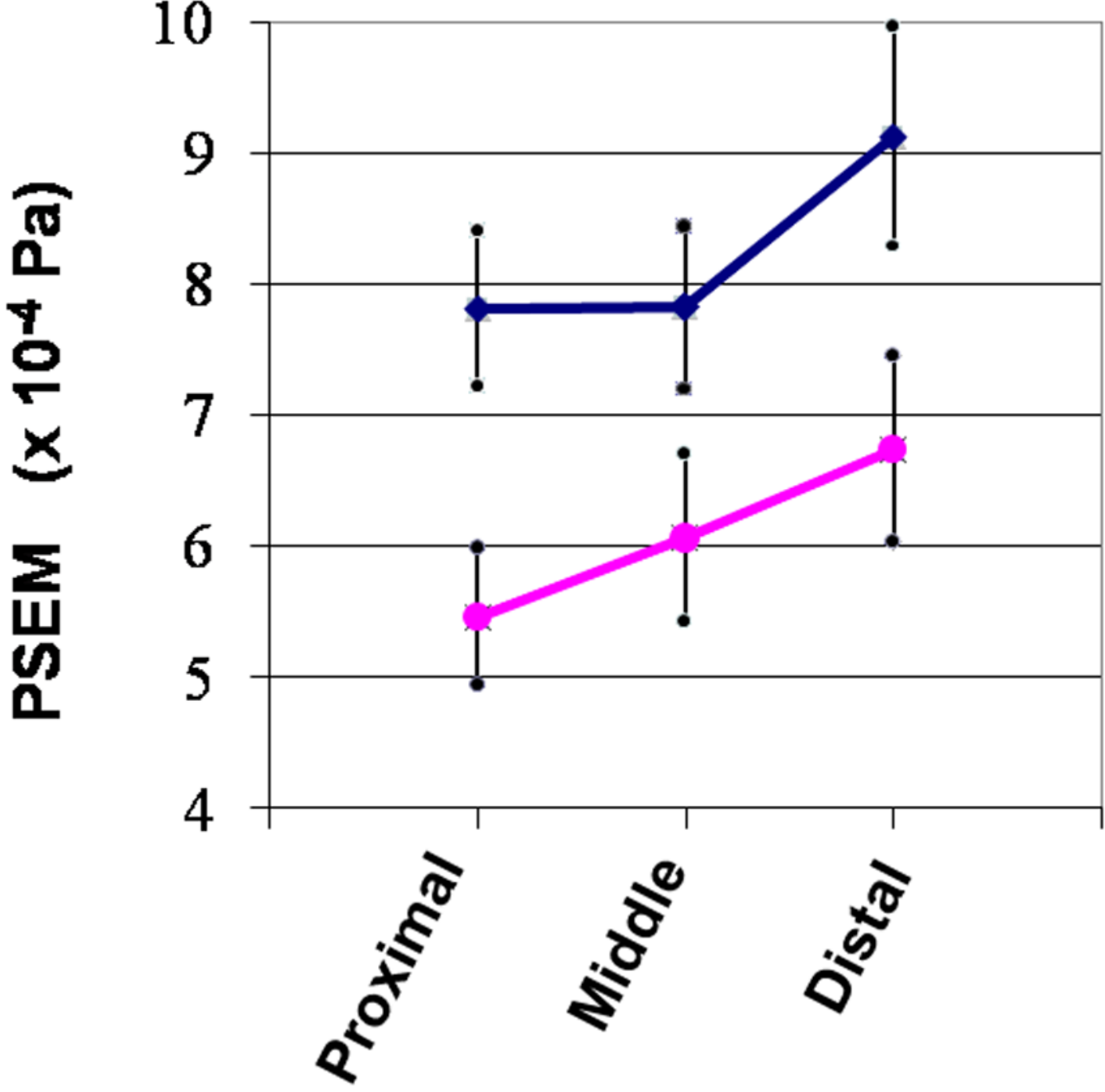

Figure 2. Pressure-Strain Elastic Modulus at the Proximal, Middle, and Distal Descending

Thoracic Aorta in Patients with SLE and Controls

Note that the pressure strain elastic modulus (PSEM, mean \pm SE) is higher in patients (blue line) than in controls (pink line) at every aortic location. Also note that PSEM progressively increases from the proximal to distal aorta. Unadjusted, $\mathrm{p}=0.002$ for group and $\mathrm{p}=0.05$ for location. Adjusted for age, $\mathrm{p}=0.02$ for group and $\mathrm{p}=0.04$ for location. 


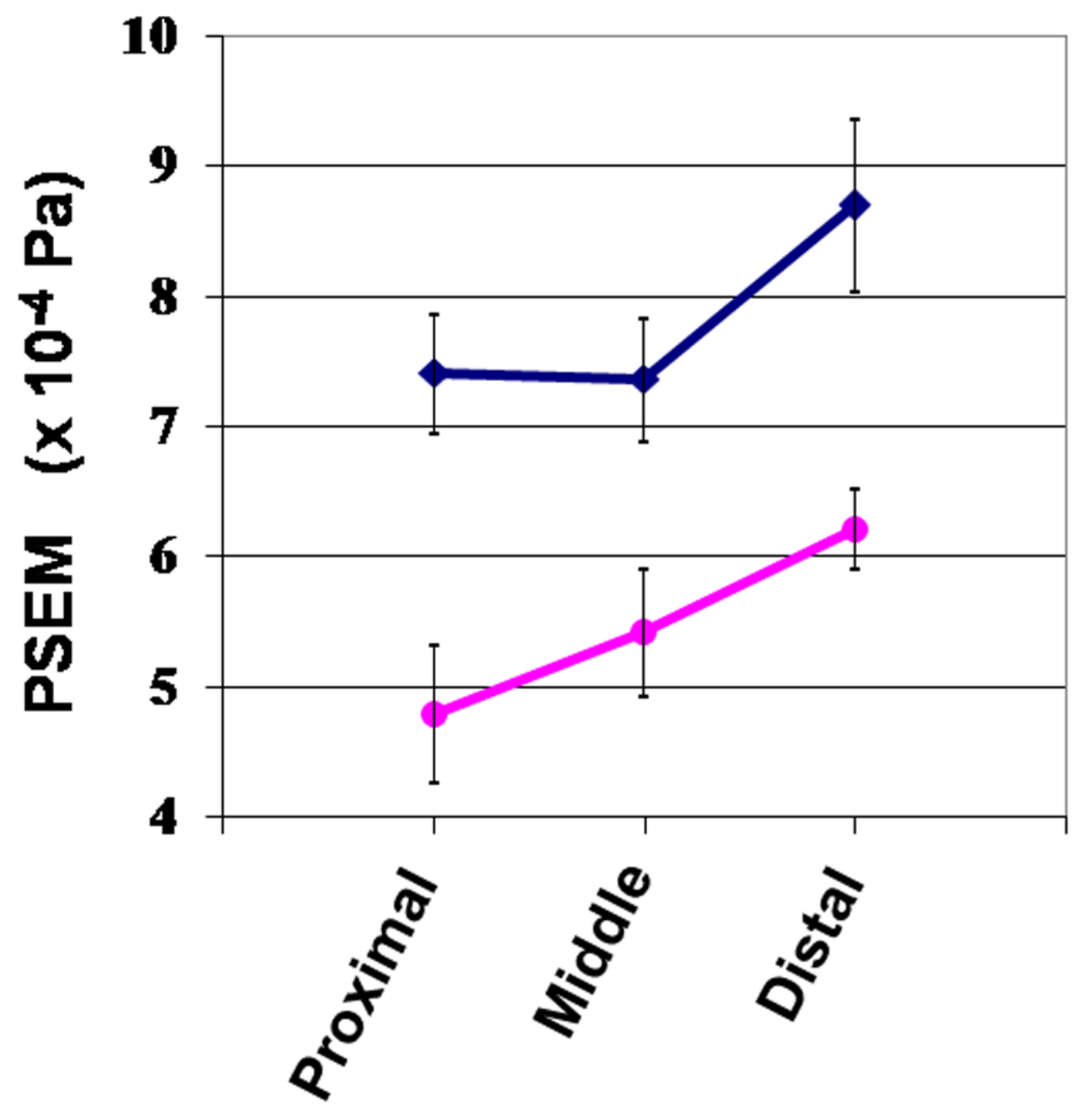

Figure 3. Pressure-Strain Elastic Modulus in Patients and Controls after Adjusting Both Groups to the Same Age, Blood Pressure, Renal Function, and Aortic Intima-media Thickness

SLE patients (blue line) had significantly higher PSEM (mean \pm SE) than controls (pink line) at every aortic location $(\mathrm{p}=0.005)$ and a linear trend up of PSEM from proximal to distal aorta was noted $(\mathrm{p}=0.04)$. 
Table 1

Clinical and Laboratory Data in Patients with SLE and Controls

\begin{tabular}{|c|c|c|c|}
\hline Parameter & $\begin{array}{l}\text { Patients } \\
(\mathbf{N}=50)\end{array}$ & $\begin{array}{l}\text { Controls } \\
(\mathbf{N}=22)\end{array}$ & P Value \\
\hline Age (years) & $38 \pm 12$ & $34 \pm 12$ & 0.15 \\
\hline Female gender $(\%)$ & 92 & 82 & 0.19 \\
\hline Non hispanic whites and Hispanic (\%) & 28 and 60 & 36 and 50 & 0.71 \\
\hline Body mass index $\left(\mathrm{kg} / \mathrm{m}^{2}\right)$ & $27 \pm 5$ & $27 \pm 6$ & 0.75 \\
\hline *Systolic blood pressure $(\mathrm{mmHg})$ & $124 \pm 14$ & $116 \pm 8$ & 0.01 \\
\hline${ }^{*}$ Diastolic blood pressure $(\mathrm{mmHg})$ & $77 \pm 10$ & $73 \pm 7$ & 0.03 \\
\hline${ }^{*}$ Mean arterial blood pressure $(\mathrm{mmHg})$ & $93 \pm 10$ & $87 \pm 6$ & 0.01 \\
\hline Ambulatory hypertension $(\%)$ & 14 & 0 & 0.09 \\
\hline Hypertension or on vasodilator therapy (\%) & 28 & 0 & 0.004 \\
\hline Smoking & 54 & 23 & 0.02 \\
\hline Cholesterol (mg/dl) & $182 \pm 45$ & $185 \pm 45$ & 0.82 \\
\hline Triglycerides (mg/dl) & $153 \pm 77$ & $160 \pm 104$ & 0.79 \\
\hline Dyslipidemia & 63 & 38 & 0.07 \\
\hline Diabetes mellitus (\%) & 12 & 0 & 0.17 \\
\hline Any atherogenic risk factor $(\%)$ & 72 & 36 & 0.01 \\
\hline Post-menopausal status (\%) & 15 & 11 & 1.0 \\
\hline Hematocrit $(\%)$ & $39 \pm 4$ & $41 \pm 4$ & 0.02 \\
\hline Platelets $\left(\mathrm{k} / \mathrm{mm}^{3}\right)$ & $240 \pm 91$ & $274 \pm 44$ & 0.04 \\
\hline White blood cell count $\left(\mathrm{k} / \mathrm{mm}^{3}\right)$ & $5.8 \pm 2.3$ & $6.5 \pm 1.7$ & 0.19 \\
\hline Creatinine (mg/dl) & $0.83 \pm 0.32$ & $0.72 \pm 0.10$ & 0.04 \\
\hline $\operatorname{Albumin}(\mathrm{g} / \mathrm{dL})$ & $3.9 \pm 0.6$ & $4.2 \pm 0.4$ & 0.01 \\
\hline P-selectin (ng/mL) & $41 \pm 21$ & $39 \pm 16$ & 0.62 \\
\hline $\mathrm{C} 3 \mathrm{a}(\mathrm{pg} / \mathrm{mL})$ & $2246 \pm 3255$ & $2096 \pm 2217$ & 0.83 \\
\hline $\mathrm{C} 5 \mathrm{a}(\mathrm{pg} / \mathrm{mL})$ & $31 \pm 11$ & $29 \pm 10$ & 0.54 \\
\hline Quantitative D-dimer (ug/ml) & $1.0 \pm 3.0$ & $0.3 \pm 0.4$ & 0.15 \\
\hline Platelet derived microparticles (uL) & $553 \pm 668$ & $517 \pm 592$ & 0.83 \\
\hline Monocyte derived microparticles (uL) & $442 \pm 668$ & $477 \pm 487$ & 0.81 \\
\hline Endothelium derived microparticles (uL) & $162 \pm 235$ & $154 \pm 122$ & 0.87 \\
\hline
\end{tabular}

Data presented as mean $\pm \mathrm{SD}$ or $\%$.

* Ambulatory blood pressure. 
Table 2

Clinical, Therapy, and Laboratory Data in Patients with SLE

\begin{tabular}{|c|c|}
\hline Characteristic & Patients $(\mathrm{N}=\mathbf{5 0})$ \\
\hline Duration of SLE (years) & $9 \pm 7$ \\
\hline Age of SLE diagnosis (years) & $30 \pm 13$ \\
\hline Total SLEDAI (U) & $12 \pm 10$ \\
\hline Non-Neuro-SLEDAI (U) & $8 \pm 6$ \\
\hline Total SLICC (U) & $3 \pm 2$ \\
\hline Non-Neuro-SLICC (U) & $2.5 \pm 1.7$ \\
\hline Prednisone therapy $(\%)$ & 42 \\
\hline Prednisone average dose (mg/d) & $8 \pm 7$ \\
\hline Prednisone (years) & $7.2 \pm 6.6$ \\
\hline Cyclophosphamide therapy (\%) & 44 \\
\hline Years of cyclophosphamide therapy (\%) & $0.7 \pm 0.9$ \\
\hline Methotrexate, azathioprine, mycophenalate, or retuximab (\%) & 52 \\
\hline Hydroxycloroquine or cloroquine therapy (\%) & 62 \\
\hline Warfarin, aspirin, or clopidogrel (\%) & 38 \\
\hline Positive DNA (\%) & 44 \\
\hline DNA titer (dilutions) & $66 \pm 236$ \\
\hline ANA titer (dilutions) & $374 \pm 455$ \\
\hline Smith antibody positive (\%) & 30 \\
\hline SSA antibody positive (\%) & 38 \\
\hline SSB antibody positive (\%) & 12 \\
\hline Ribonucleoprotein antibody positive (\%) & 22 \\
\hline Ribonucleoprotein titer (dilutions) & $4.7 \pm 20$ \\
\hline $\mathrm{C} 3(\mathrm{mg} / \mathrm{dl})$ & $99 \pm 33$ \\
\hline $\mathrm{C} 4(\mathrm{mg} / \mathrm{dl})$ & $20 \pm 25$ \\
\hline CH50 (mg/dl) & $81 \pm 37$ \\
\hline C-reactive protein $(\mathrm{mg} / \mathrm{dl})$ & $1.1 \pm 1.5$ \\
\hline Erythrosedimentation rate $(\mathrm{mm} / \mathrm{hr})$ & $25.4 \pm 25.2$ \\
\hline Antiphospholipid antibody positive (\%) & 60 \\
\hline IgM anticardiolipin antibody (IU) & $10 \pm 15$ \\
\hline IgG anticardiolipin antibody (IU) & $13 \pm 20$ \\
\hline IgA anticardiolipin antibody (IU) & $6 \pm 12$ \\
\hline Beta 2 glycoprotein antibody positive (\%) & 23 \\
\hline Lupus-like inhibitor positive (\%) & 33 \\
\hline
\end{tabular}

Data presented as mean $\pm \mathrm{SD}$ or $\%$.

SLEDAI = SLE disease activity index; SLICC = SLE International Collaborating Clinics Damage Index; DNA = deoxyribonuclease antibody; ANA $=$ antinuclear antibody. 


\section{Table 3}

Aortic Pressure-Strain Elastic Modulus (Pascal Units) in Patients and Controls

\begin{tabular}{|c|c|c|c|}
\hline Location & Patients $(n=50)$ & Controls $(n=22)$ & P-value \\
\hline Proximal & $7.81 \pm 4.08$ & $5.46 \pm 2.46$ & 0.004 \\
\hline Mid aorta & $7.82 \pm 4.24$ & $6.06 \pm 2.99$ & 0.05 \\
\hline Distal aorta & $9.13 \pm 5.56$ & $6.74 \pm 3.31$ & 0.03 \\
\hline \multirow[t]{2}{*}{ Overall } & $8.25 \pm 4.13$ & $6.1 \pm 2.5$ & 0.01 \\
\hline & *Normotensive TEE $(\mathrm{N}=41)$ & * Normotensive TEE $(\mathrm{N}=20)$ & \\
\hline \multirow[t]{4}{*}{ Overall } & $7.4 \pm 3.2$ & $5.8 \pm 2.2$ & 0.03 \\
\hline & ${ }^{*}$ Ambulatory normotensive $(\mathrm{N}=37)$ & *Ambulatory Normotensive $(\mathrm{N}=22)$ & \\
\hline & $7.9 \pm 4.4$ & $6.1 \pm 2.5$ & 0.04 \\
\hline & No aortic plaques $(\mathrm{N}=36)$ & No aortic plaques $(\mathrm{N}=22)$ & \\
\hline \multirow[t]{2}{*}{ Overall } & $7.8 \pm 4$ & $6.1 \pm 2.5$ & 0.046 \\
\hline & Non-smokers $(\mathrm{N}=23)$ & Non-smokers $(\mathrm{N}=17)$ & \\
\hline Overall & $8.98 \pm 4.5$ & $6.0 \pm 2.2$ & 0.01 \\
\hline
\end{tabular}


Table 4

Predictors of Aortic Stiffness in Patients with SLE

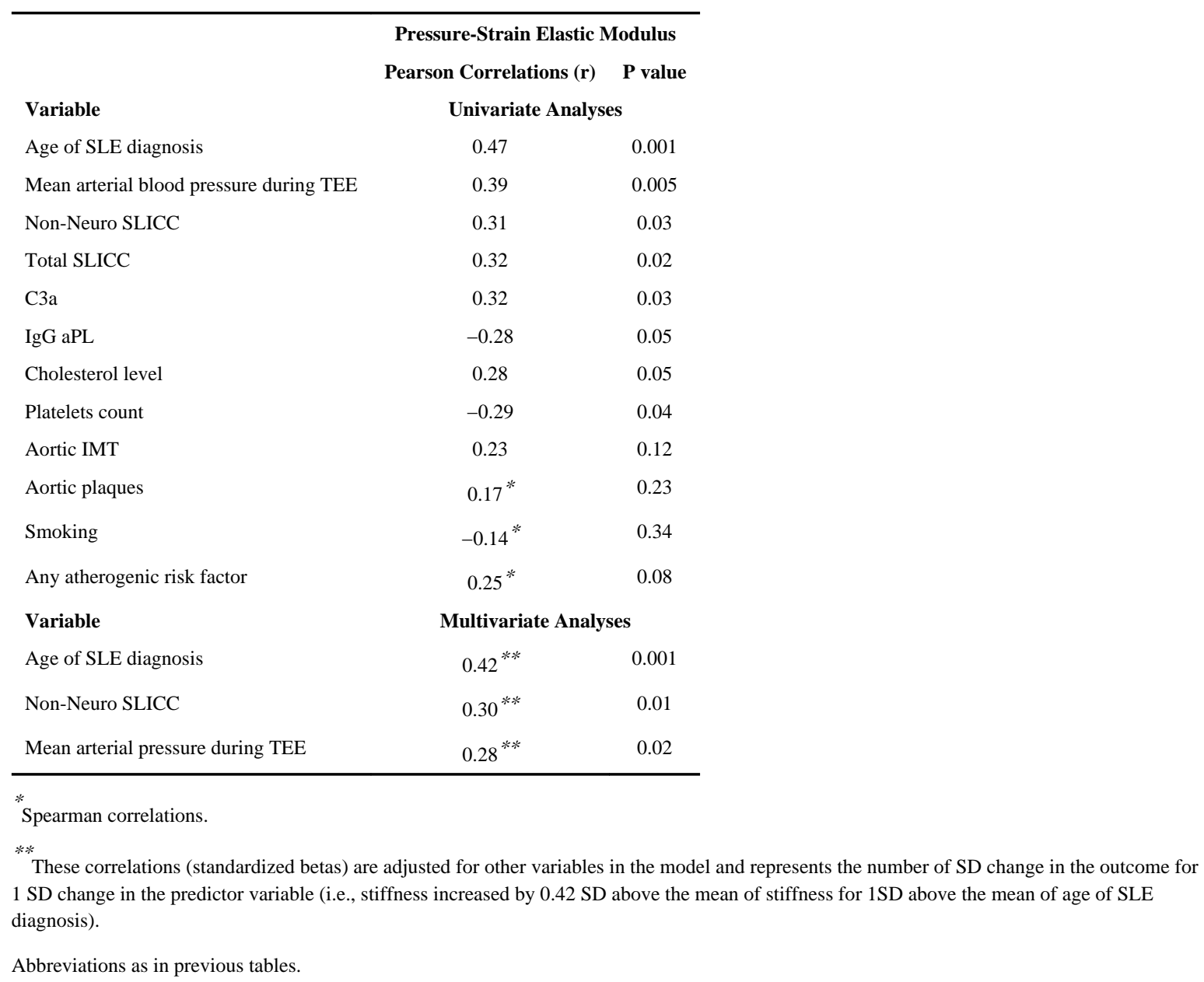

